\title{
Journal of Physics C \\ Solid State Physics
}

\section{Letters to the Editor}

\section{Energy gaps in amorphous covalent semiconductors}

\begin{abstract}
A calculation of approximate density of states for a disordered covalent semiconductor shows that the energy gap is due to the presence of short range order.
\end{abstract}

It is difficult to explain the persistence of an energy gap when a covalent semiconductor is prepared in the amorphous state (Ziman 1969). Without long range crystalline order the usual concepts in band theory are meaningless, and the analysis must be based upon the local structural order-for example, 'tetrahedral glass' configurations proposed by Grigorivici (1969) for amorphous Ge. In this letter it is shown that the approximate density of states for a model system of carbon atoms with this structure has an energy gap very similar to the energy gap of crystalline diamond.

The calculation depends upon a convenient feature of multiple scattering theory-an expression for the density of states may be written down in terms of the location of the sites and scattering properties of each site. This expression may be rearranged so that the overall scattering properties of a cluster of atoms replace those of a single atom, making possible the study of the effect of short range order. Neglecting the scattering between clusters, we may write down the following approximate formula for the density of states, at energy $E$, (Lloyd 1967)

$$
\rho(E)=\frac{E^{1 / 2}}{2 \pi^{2}}-\frac{2}{\pi \Omega} \frac{\mathrm{d}}{\mathrm{d} E} \sum_{j} \operatorname{Im} \operatorname{Tr} \ln \left\{\delta_{L L^{\prime}}+\mathrm{i}(E)^{1 / 2} K_{L L^{\prime}}(j)\right\} .
$$

The important term in this expression is the reaction matrix $K_{L L^{\prime}}(j)$ of the $j$ th cluster in the angular momentum representation. For a cluster of muffin-tin wells, this can easily be evaluated numerically by the usual devices of scattering theory.

The important clusters in amorphous $\mathrm{Ge}$ and $\mathrm{Si}$ are of eight atoms in two tetrahedra bonded together in the 'staggered' or 'eclipsed' configurations. To test the method, we have used the atomic scattering properties ( $\mathrm{s}$ and $\mathrm{p}$ phase shifts) of carbon which produce a large band gap in the crystalline state, approximating very well the known band structure of diamond. A weak resonance in the p-wave scattering plays an important role in the results reported here and seems to be essential to obtaining a gap even in the perfect crystal. (R. Gilbert and G. J. Morgan, to be published, Klima and McGill, to be published).

Figure 1 shows the density of states calculated from equation (1) for the two different types of clusters together with the position of the $\Gamma_{25}^{\prime}$ and $\Gamma_{15}$ band edges in the crystal. 
The steep drop in $\rho(E)$ at about $0.5 \mathrm{ryd}$, and the rise at 1.0 ryd are very close to the top of the valence band and the bottom of the conduction band, respectively; thus, there is a low density of states over a range of energy coinciding with the energy gap. The peaks in $\rho(E)$ on either side derive from the p-resonances which are split into two groups by interactions between the sites in a cluster. Whilst equation (1) is only an approximation to the

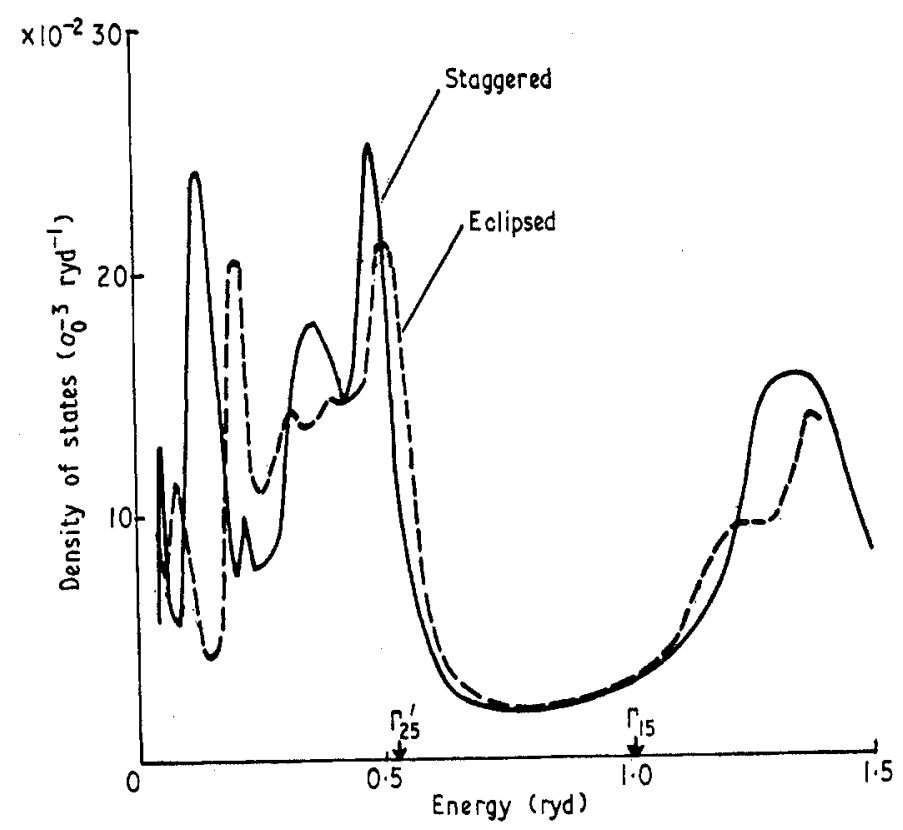

Figure 1. The density of states as a function of energy for solids composed of the staggered and eclipsed structures.

true density of states, the similarity of the two curves for the staggered and eclipsed local configurations suggests that the energy gap for a tetrahedral glass should be very similar to the energy gap in the corresponding crystal-in full agreement with experiment (Stuke 1970).

We are now undertaking a more detailed calculation with phase shifts typical of Si and $\mathrm{Ge}$, where the small band gap sets a much more severe test of the idea. Preliminary indications are that the scattering properties of these atoms-including weak p-resonancesare so similar to those of carbon that the same phenomena should be observed. A complete account of these investigations will be submitted for publication in this journal in due course.

We are grateful to a NATO Fellowship (TCMcG) and to a grant from the Ministry of Technology $(\mathrm{JK})$ for financial support. We gratefully acknowledge the continuing guidance of Professor J. M. Ziman.

H. H. Wills Physics Laboratory

T. C. MCGILL University of Bristol

Grigorovici, R., and MANAILA, R., 1969, J. Noncryst. Solids, 1, 371-87.

Lloyd, P., 1967, Proc. Phys. Soc., 90, 207-16.

STuke, J., 1970, J. Noncryst. Solids, 4, 1-26.

Zrman, J. M., 1969, J. Phys. C: Solid St. Phys., 2, 1704-16. 